\title{
SOLARNET \& LAIME: Imaging \& Spectroscopy in the Far Ultraviolet
}

\author{
Luc Damé ${ }^{1,2}$ and Serge Koutchmy ${ }^{3}$ \\ ${ }^{1}$ Service d'Aéronomie du CNRS, BP 3, 91371 Verrièrres-le-Buisson Cedex, France \\ email: luc.dame@aerov.jussieu.fr \\ ${ }^{2}$ LESIA, Observatoire de Paris-Meudon, 5 place Jules Janssen, Meudon 92195, France \\ email: luc.dame@obspm.fr \\ ${ }^{3}$ Institut d'Astrophysique de Paris, 98bis boulevard Arago, 75014 Paris, France \\ email: koutchmy@iap.fr
}

\begin{abstract}
SOLARNET is a medium size high resolution solar physics mission proposed to CNES and ESA for a new start in 2007 and a possible launch in 2012 (CNES) or later (ESA Cosmic Vision framework: 2015-2016). Partnerships with India and China are under discussion, and several European contributions are considered. At the center of the SOLARNET mission is a 3-telescope interferometer of 1 meter baseline capable to provide 40 times the best ever spatial resolution achieved in Space with previous, current or even planned solar missions: 20 mas - $20 \mathrm{~km}$ on the Sun in the FUV. The interferometer is associated to an on-axis Subtractive Double Monochromator coupled to an Imaging Fourier Transform Spectrometer capable of high spectral $(0.01 \mathrm{~nm})$ and high temporal resolutions $(50 \mathrm{~ms})$ on a field of view of $40 \operatorname{arcsec}$ and covering the FUV and UV spectral domains (from 117.5 to $400 \mathrm{~nm}$ ). This will allow to access process scales of magnetic reconnection, dissipation, emerging flux and much more, from the chromosphere to the low corona with emphasis on the transition zone where the magnetic confinement is expected to be maximum. A whole new chapter of the physics of solar magnetic field structuring, evolution and mapping from the photosphere to the high atmosphere will be opened. The interferometer is completed by instruments providing larger field of view and higher temperature (EUV-XUV coronal imaging \& spectroscopy) to define the context and extension of the solar phenomena. The 3-telescope interferometer design results of an extensive laboratory demonstration program of interferometric imaging of extended objects. We will review the scientific program of SOLARNET, describe the interferometer concept and design, present the results of the breadboard and give a short overview of the mission aspects. In a different category, LAIME, the Lyman Alpha Imaging-Monitor Experiment, is a remarkably simple (no mechanisms) and compact full Sun imager to be flown with TESIS on the CORONAS-PHOTON mission in 2008. It could be the only chromospheric imager to be flown in the next years, supporting Solar-B, STEREO, SDO and the Belgian LYRA Lyman Alpha flux monitor. We will give a short description of this unique $60 \mathrm{~mm}$ aperture imaging telescope, dedicated to the investigation of the UV sources of solar variability and of the chromospheric and coronal disruptive events (Moreton waves, prominences, CMEs, etc.).
\end{abstract}

Keywords. Sun, interferometer, high resolution, transition region, chromosphere, magnetic fields, UV, FUV.

\section{Introduction \& Scientific Rationale}

The mission we propose is to provide, at high spatial, spectral and temporal resolutions, observations of the solar atmosphere and new insights of the solar interior. Novel observations would be made by SOLARNET, a cophased interferometer of 1 meter baseline coupled to a UV double monochromator to join high spatial, temporal and spectral 
resolutions. By using interferometry in the UV with a spectro-imaging mode SOLARNET will allow remote sensing of the solar surface and atmosphere with an unprecedented spatial resolution of $20 \mathrm{~km}$ on the Sun that Solar Probes or Orbiters, despite their closer distance to the Sun, will never achieve. SOLARNET is the proper success combination of the spectral completeness of SOHO and the UV, FUV imaging provided by TRACE. Since the plasma confinement and acceleration processes are most probably in the high chromosphere and transition zone, this FUV imaging (and doppler-imaging) is the most appreciable - beside high resolution - enhancement compared to SOHO. The mission, like SOHO, has also a permanent Sun viewing orbit which allows resolved and global helioseismology. This is the second breakthrough of the proposed mission, its unique package of helioseismology instruments for diameter oscillations (larger g-modes sensitivity in the UV), intensity, global and resolved observations, and high resolution full Sun velocity oscillations (with the MOF, Magneto Optical Filter). The third breakthrough is the thorough set of diagnostics to study the solar variability and Space weather aspects. A new set of solar constant global and spectrally resolved irradiance monitors are implemented coupled with an enhanced full sky Lyman $\alpha$ imaging and a unique solar shape, solar differential rotation telescope (NSSOT, the New Solar Shape and Oscillations Telescope). To this third set, the MOF brings the magnetograms information. This will directly address the nature of UV variability and its climate consequences. EUV and $\mathrm{X}$-ray imagers and spectrometers and an UV/visible coronagraph complete the payload. This mission will achieve its impressive aims with a relatively small and simple spacecraft on a common reusable small platform on low-Earth orbit $(1000 \mathrm{~km})$, novelty being in state of-the-art, new instruments allowing the use of such a small platform and, consequently, an affordable launcher. The several new technologies of the mission will lead to new insights into how the Sun works from the interior to the corona and to the Earth. With orders of magnitude in spatial resolution but also in sensitivity, it has the potential to resolve major open questions in solar physics and to bring in important discoveries.

\section{Model Payload}

Heritage of instruments from Yohkoh, SOHO, SPDE/TRC, TRACE and VAULT was used as a baseline for designing the payload. Instruments are state-of-the-art and, thanks to the low-Earth orbit and the mini-satellite class of the platform - still allowing a 350 to $500 \mathrm{~kg}$ payload -, they can deliver continuous coverage of the Sun at high telemetry rate. The major instrument, the SOLARNET interferometer, makes a clever use of mass and is naturally compact and integrated in design. Even though, a large effort is made on onboard data compression/storage. Also, the use of a separated small pointing platform for the Sun centered instruments (helioseismology and coronagraphs) simplifies the design of these instruments still bringing in only a small mass (the hexapod platform with its six actuators whose extension is limited to accommodate a few degrees of pointing range). The payload includes both imaging and spectroscopy. Imagers, beside the very high resolution of the interferometer, aim at high spatial (0.1-0.2") and temporal (0.1$0.2 \mathrm{~s})$ resolutions, to reveal the context of the small-scale dynamical processes in the atmosphere and to study the rapid changes in morphology associated with transition region and coronal magnetic activity. An invaluable advantage of the low-Earth orbit is that the relative motion between the solar structures can be limited by very high temporal resolution, ensuring that the images will not be blurred during the exposure time.

The payload will consists in 8 to 10 instruments according to the launcher capacities (Eurockot or the new ESA VEGA small launcher). The SOLARNET three-telescopes 
cophased interferometer with its far UV and UV Imaging Spectrometer (with lines from chromosphere to corona), an X-ray/EUV full-Sun imager, a high-resolution EUV imaging spectrometer $(10 \mathrm{kK}-2 \mathrm{MK})$, a high-resolution visible telescope, magnetograph and velocity oscillations (MOF), an EUV and visible-light coronagraph, which has the capability to measure coronal plasma outflow velocity through Doppler-dimming of the HI $121.6 \mathrm{~nm}$ and He II $30.4 \mathrm{~nm}$ lines, and to provide UV and visible-light global images of the corona, NSSOT (New Solar Shape and Oscillations Telescope), NG-DIARAD/VIRGO (solar constant and intensity oscillations), and NG-SWAN (full sky Lyman $\alpha$ ). To these, a very high spectral resolution spectrograph might be added with a spectral resolution superior to 5000 to allow Doppler velocity determinations to better than a few $\mathrm{km} / \mathrm{s}$. It will use a novel two element, normal incidence optical design with highly reflective EUV coatings to access a spectral range with broad temperature coverage (0.03-15 MK) and density-sensitive line ratios (this spectrograph is inherited from the NRL VAULT rocket effort and the new VERIS spectrograph). Critical issues like heat ingress into the telescopes, aperture locations, mechanical structures of the instruments, have been evaluated and proven solution implemented.

The platform is of the LEOSTAR or PROTEUS type, 3-axis stabilized ( 1 arcsec) and always Sun-pointing. Platform and instruments' accommodation are illustrated on Fig. 1. An EuRockot or VEGA launcher is largely sufficient with such a small platform and can easily lift almost one ton to a Sun synchronous $1000 \mathrm{~km}$ orbit 6:00-18:00 minimizing eclipses (a few minutes 50 days or so per year; acceptable even for critical g-modes detection since sweeping). Telemetry, with a simple X-band antenna, allows impressive average downlinks superior to $3 \mathrm{Mbits} / \mathrm{s}$. The expected volume and format of the data coming from the various instruments will be orders of magnitude higher than SOHO and TRACE or any previous Solar Physics spacecrafts but small compared to current Earth Observation Missions. We therefore anticipate no particular difficulties to handle, process and archive data in an operation facility in Europe.

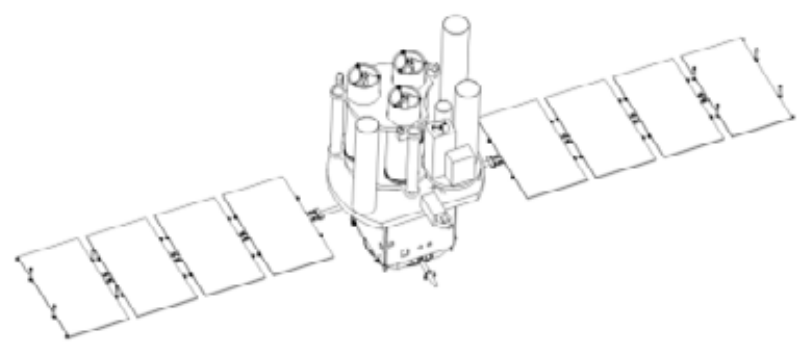

Figure 1. The 8 instruments of the SOLARNET Mission accommodated on the ALCATEL PROTEUS platform. The payload size is $\varnothing 1.7 \mathrm{~m} \times 1.8 \mathrm{~m}$. Note the small platform of $\varnothing 60 \mathrm{~cm}$, beside the 3-telescope SOLARNET interferometer, mounted on 6 linear actuators (hexapod) and accommodating the helioseismology package and coronagraphs (instruments needing to be pointed to Sun center permanently). The two "larger tubes" are the EUV imager and spectrometer and the small instrument in front, slightly pointing outside the platform base, is the NG-SWAN instrument. The VERIS spectrograph is not shown but could easily be adapted.

SOHO, complemented by TRACE, has been a very successful mission addressing all aspects of solar and heliospheric physics and, consequently, pointing out the areas in need for a better and deeper understanding. Future progress are to be expected in high resolution particularly at the level of chromospheric and transition zone imaging where the magnetic confinement and essential structuration take place. Both spatial, spectral and temporal resolutions are necessary to follow the evolution of structures and of the 
magnetic configurations. SOLARNET, the mission as a whole, is that unique combination of SOHO and TRACE.

The proposed mission is a further development of the SIMURIS (Solar, Solar System and Stellar Interferometric Mission for Ultrahigh Resolution Imaging and Spectroscopy) and SPI (Solar Physics and Interferometry) missions. More information on the payload and the different instruments proposed on SPI can be found in Damé and Derrien (2002).

\section{High Resolution UV and FUV Spectral Imaging}

With the interferometer, high-resolution imaging of the solar atmosphere will be better than in past missions by more than an order of magnitude. This will enable us to analyze thoroughly the time-variability, evolution and fine-scale structure of the dynamic chromosphere, transition region and corona, to study fully the Sun's magnetic activity on multiple scales, to investigate energetic particle acceleration, confinement and release, and to reveal plasma and radiation processes underlying the heating of the chromosphere and corona.

Table 1. Characteristics (spatial and spectral) of SOLARNET focal plan spectro-imaging instrument.

\begin{tabular}{c|c|c|c|c}
\hline $\begin{array}{c}\text { Wavelength } \\
\text { range }(\mathrm{nm})\end{array}$ & $\begin{array}{c}\text { Field-of-view } \\
(\operatorname{arcsec})\end{array}$ & $\begin{array}{c}\text { CCD dimensions } \\
(\text { pixels })\end{array}$ & $\begin{array}{c}\text { Spatial resolution } \\
(\operatorname{arcsec})\end{array}$ & $\begin{array}{c}\text { Spectral } \\
\text { resolution }(\mathrm{nm})\end{array}$ \\
\hline $\begin{array}{c}117-200 \\
130-300\end{array}$ & $\begin{array}{c}40 \times 40 \\
60 \times 60\end{array}$ & $2048 \times 2048$ & $\begin{array}{c}0.025-0.04 \\
0.04-0.1\end{array}$ & $\begin{array}{c}0.1\left(0.002^{*}\right) \\
\sim 8\end{array}$ \\
\hline $280-400$ & $60 \times 60$ & $2048 \times 2048$ & $0.06-0.08$ & $0.02\left(0.001^{*}\right)$ \\
\hline
\end{tabular}

* This could be achieved with an extra filtering (Fabry-Perot or IFTS)

The relevant minimum observable scale in the solar atmosphere may be of the order of 3-20 km since smaller scales will probably be smeared out by plasma micro-instabilities (such as drift waves). This scale range is smaller though comparable to the photon mean free path in the chromosphere $(50-70 \mathrm{~km})$. Slightly larger scales can be expected in the corona (though gradient across coronal loops may also be a few $\mathrm{km}$ ). Altogether this situation is rather fortunate because we have access to higher resolutions in the far UV than in the visible and X-rays (multilayer telescopes are limited to resolutions of $0.1-$ 0.2 arcsec or so). In the UV, the emission lines are generally thin, i.e. not affected by the optically thick transfer conditions (which prevail in the visible and near UV lines accessible from ground) and we can expect to see structures with scales 3 to $30 \mathrm{~km}$. This was observed by HRTS (the NRL High Resolution Telescope and Spectrograph) in Si IV and C IV lines for example by the filling factors deduced from high resolution spectra, cf. Dere et al. (1987) and Brekke (1999). In the visible, thick transfer conditions blurs the signature of structures and nothing smaller than the 50-70 km limit should be observed. This means that with a single instrument of meter class diameter we have the appropriate, scientifically justified, spatial resolution for both the far UV $(20 \mathrm{~km}$ in the C III line $117.5 \mathrm{~nm}$ ) and the near UV (60 km in the Ca II K line $\lambda 396.3 \mathrm{~nm}$ ).

A breakthrough in high spatial resolution observations (20 km is 40 times more spatial resolution than any previous solar instrument in Space) should allow to understand in finer physical details processes like magnetic heating in coronal loops (temperature profiles, time dependence, spatial local ionization of heating processes-are loops bundles of very small unresolved strands that are heated quasi-randomly, not at the same time, like suggested in Klimchuk 2006?) but, also, by access to visible wavelengths, the coupling 
between turbulent convective eddies and magnetic fields in the photosphere. Another scientific objective is the plasma heating processes and thermal inputs of flares and microflares and their fine magnetic field structures. Heating, flares and microflares but also internal structure (g-modes) are "big" questions that indeed, after years of limited observations, could now be addressed.

Answers to the questions require the study of magnetic flux elements on their intrinsic spatial scale. The high-resolution of the imagers, spectro-imagers and magnetograph is intended to monitor the emergence, dynamics, twist, shearing, mutual interactions and possible coalescence and subduction below the surface in order to follow the evolution and scrutinize the life cycles of magnetic flux elements. Coupled with the resolved oscillations of the MOF, the rotation below the surface can be addressed and, accordingly, the relation between convection and magnetism.

This very high resolution need is fulfilled by novel instrumentation: a 3-telescope cophased interferometer of meter class feeding a high resolution imaging spectrometer. The important technologies needed by the proposed interferometer concept include optical delay lines and a monitoring and cophasing system together with its control software. These technologies have noticeably been breadboarded successfully by Service d'Aéronomie (Damé et al. 2004; Damé 2003; Damé et al. 2002). Experiments have worked out extremely well and all components needed by an interferometer have been validated through an end-to-end test from object to image reconstruction. These achievements secure the development of the SOLARNET interferometer as both details and system design issues are perfectly mastered. The working three telescopes solar imaging interferometer is currently in use and visible at the "Grand Sidérostat de Foucault" at Meudon Observatory. And, after completion of an optimized fine pointing for the three telescopes this spring (cf. Fu et al. 2006) and providing an excellent 0.1" pointing stability, the first interferometric direct images of the Sun should be obtained this summer (note that imaging with 3 telescopes on extended artificial sources was achieved in laboratory since 2000, cf. Damé et al. 2002). Note that the hearth of the cophasing system, the three reference interferometers that measure the phase in white light, has been spatialized as a compact block of reduced dimensions $(5 \times 5 \times 15 \mathrm{~cm})$ through molecular binding (see ?, for example)DamCla].

The principal characteristics of the major instrument, the high-resolution spectroimager, are presented in Table I. This instrument consists in two subtractive double monochromators (SDM) coupled through the use of a Wadsworth relay mirror. The system has been described in several papers, e.g. in Damé et al. (2004) and references herein. Recently, we further optimized the design of the SDM to access even larger field of view with maximum spectral resolution. For this (cf. Fig. 2) we make a clever use of the hollow pupil, going through it with the reconstituted beam, what allows the use of on-axis optics which minimize aberrations. With such a design, $0.1 \mathrm{~nm}$ spectral resolution is possible on the full 40 arcsec FOV. To achieve even higher spectral resolutions and to deliver a complete line profile, an extra filtering stage would be required, e.g. a Fabry Perot or an Imaging Fourier Transform Spectrometer (IFTS). In this case, the Double Monochromator acts as a pre-filter to limit the input bandwidth to the free spectral interval, $\sim 8 \mathrm{~nm}$ (for $0.002 \mathrm{~nm}$ resolution). We first started studying an IFTS in the framework of the SIMURIS ESA mission Phase A (see Thorne 1992, for example), and this effort was pursued by P. Lemaire and resulted in a promising breadboard (Millard et al. 2005). A tunable Fabry Perot would also be possible, like the one we developed 20 years ago to observe the C IV lines in the framework of a SPARTAN flight. 


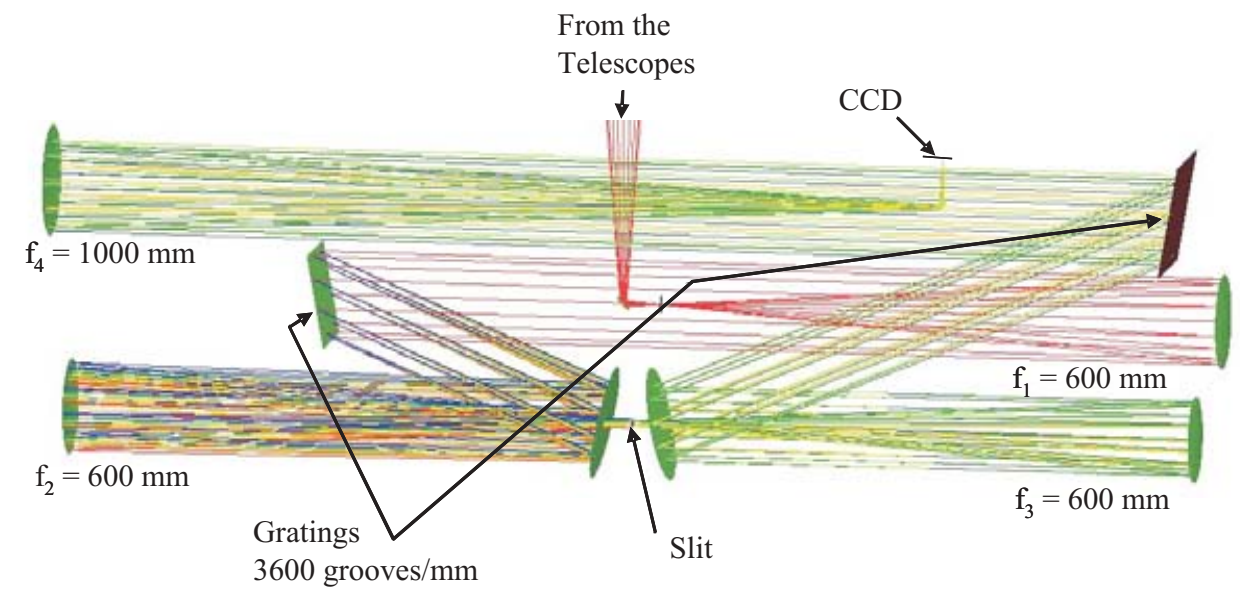

Figure 2. New configuration of the spectro-imaging focal plane instrument of SOLARNET. Note that the relay optics are now on-axis what minimizes the aberrations. In output the reconstituted beam can be focused on the CCD or collimated towards an IFTS or Fabry-Perot.

\section{LAIME science}

While SOLARNET is a mission devoted to high resolution, LAIME is certainly one of the small complimentary instruments that provide context information. Coupled to a Lyman Alpha coronagraph (like in the LYOT instrument) it is an excellent context instrument to provide the topology and dynamics of the magnetic field in the transition zone at the crucial interface between the upper chromosphere and corona.

As an "independent" instrument associated to TESIS/CORONAS, the prime objective of LAIME is the measurement of the solar variability of magnetic origin, in the context of the study of the influence of the Sun on the climate of the Earth. HI Ly $\alpha$ is indeed measured since 1997, in particular by UARS and EOS/SOLSTICE, and it will soon be also by the Belgian experiment LYRA on the PROBA-2 micro-satellite of ESA (launch expected in September 2007). However, as these experiments measuring irradiance see the Sun like a star, they do not have information on the physical causes of the observed changes of irradiance. To identify the causes of these changes and measure the parameters according to solar magnetism, an imager of the whole disc with a fair spatial resolution and a good rate of observation is necessary. The nature of the variations of Ly $\alpha$ irradiance is also significant to interpret the variations of ozone and the formation of the layer-D of the ionosphere. Moreover, photometric images in Ly $\alpha$ (including their running subtraction) will show phenomena of coronal significance, such that fast waves (Moreton waves, etc.) which are propagating close to the surface and produce a signature on the structures of the chromosphere. It will be also possible to study the eruption of prominences and abrupt disappearances of filaments with a very great sensitivity and, undoubtedly, to detect phenomena of waves associated with large-scale coronal instabilities, obviously related to CMEs. The great sensitivity to the variations of temperatures of Ly $\alpha$ and the relative insensitivity to Doppler-Fizeau effects of the flux of the line (compared to $\mathrm{H} \alpha$ ) become, then, an advantage. Lastly, on the disc, the images will make it possible to apprehend the still mysterious "EUV-filament channels" and their dynamics, with possibly a faint dimming related to the CME phenomenon.

Ly $\alpha$ is the most intense solar emission line. This line is obviously very sensitive to the temperature variations in the chromosphere, but also to velocities and to magnetic fields (Zeeman effect). It is optically much thicker than the $\mathrm{H} \alpha$ line (see models by 
P. Gouttebroze and J-C Vial, for example). Thus, the "cold" structures of the corona that are apprehended, like on the first photographic images of the whole disc made by the French TRC experiment (then SPDE) on sounding rockets (R.M. Bonnet, L. Damé and collaborators). These images, already old (the firsts go back to 1979, cf. Fig. 3), remain still the best for the full Sun (resolution: 1 second of arc) to evaluate the structuring on the whole disc; they constitute an invaluable heritage for LAIME (LAIME uses many of the TRC successful technical approaches).

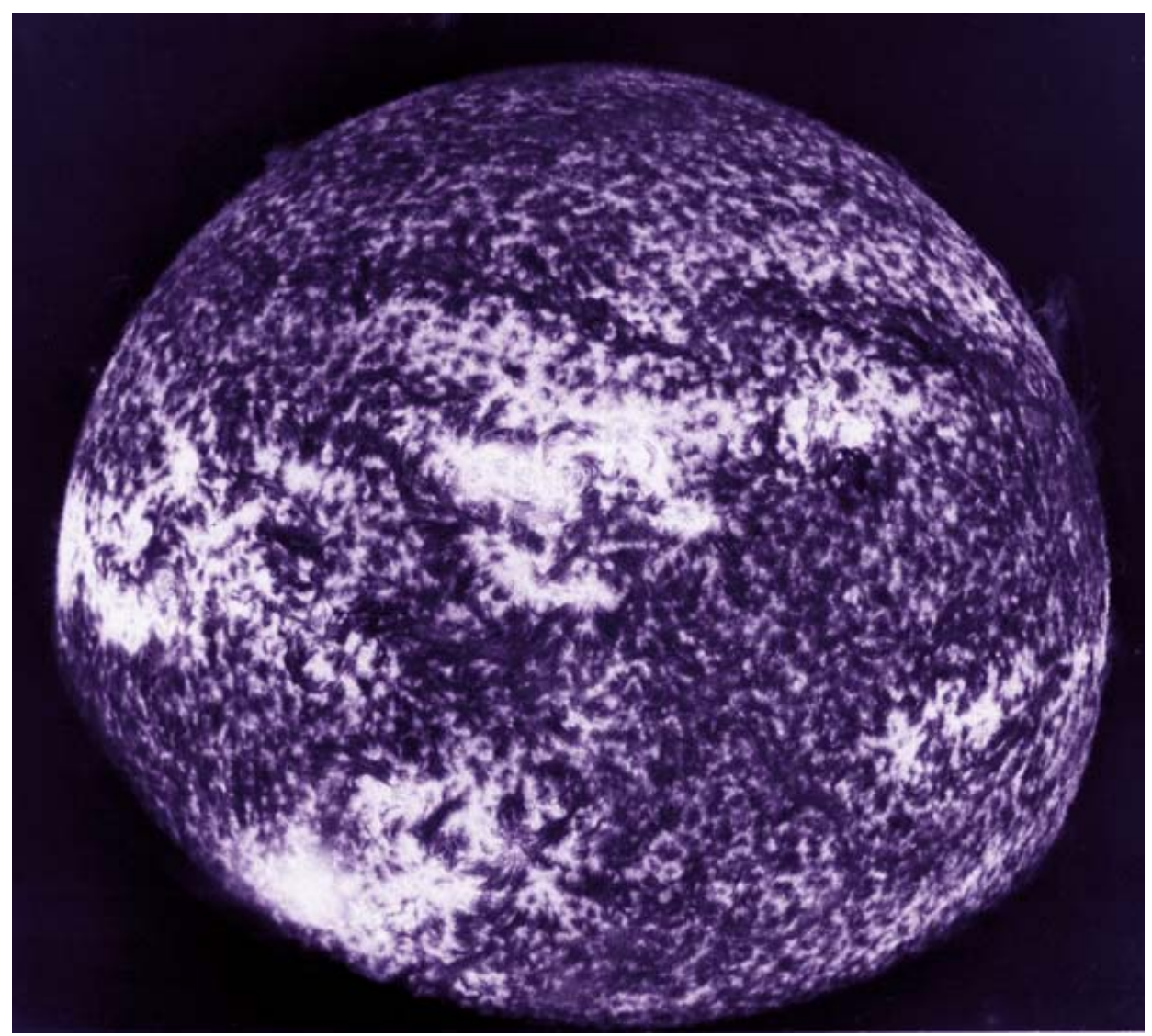

Figure 3. TRC/SPDE filtergram in Lyman Alpha $(121.6 \mathrm{~nm})$ obtained during the first rocket flight in 1979. Note the loops at the limb as well as the prominences visible even with a good exposure of the disc (and a dynamics of the film lower than that of the CCD). The high spatial resolution ( 1 arcsec) resolution explains the high contrast observed. Ly $\alpha$ is a remarkable tracer (the best) of the solar activity in the chromosphere and the low corona (the slight vignetting observed results from pointing to the large active region rather than to the Sun center).

Access to the study and monitoring of the high chromosphere crossed by all the magnetic field responsible for the active solar "dynamics", is possible since Ly $\alpha$ is precisely formed in these layers. The filtergrams obtained in this line show structures (dark: filaments; fibrils; penumbral filaments of the sunspots, etc.; and bright: plages; faculae; flares; brilliant points; prominences, etc.) which are excellent tracers of the magnetic field at the base of the corona, because all the phenomena of heating at these altitudes are due to the dissipation of the magnetic field, producing a good correlation with structures, including their alignment. Around the disc, the "cool" structures of the corona, up to very high altitudes, are visualized in an ideal way. 
Only the photospheric magnetic field is quantitatively measurable today; it reveals only the "roots" of the magnetic activity, that related to the photospheric layers. The disadvantage is that this measured field is formed in the dense layers of the photosphere (altitude of approximately $150 \mathrm{~km}$ ), on a thickness (of approximately $100 \mathrm{~km}$ ) very limited compared to the thickness of the layers crossed during the emergence of the field, those which will play a determining role in the activity of the corona. Knowledge, starting from current measurements, from what occurs in the thickness (at least on $2000 \mathrm{~km}$ ) from the non yet coronal solar atmosphere, is thus very limited. Higher, the dynamic processes dominate the transition layers, producing a mixture of cold and hot structures partly described like a phenomenon of turbulence. The emerged field in the chromosphere is impossible to calculate correctly by extrapolating the photospheric field through all this thickness where so much things are taking place, whatever the degree of sophistication of the programs used; however, the field that actually emerges, determines all the phenomena of activity of the corona.

Moreover, the "dynamical" process of emergence is badly known and extraordinarily complex, because the phenomena of small scale are closely dependent between them and play various roles according to their size. It is enough to quote the famous spicules, of which the dynamic structure is still mysterious and whose origin is still unknown, even if related to the magnetic network of the super-granulation (a definite field of stationary and horizontal velocities which extends on cells from approximately $30000 \mathrm{~km}$ diameter, cf. Filippov and Koutchmy 2000). The magnetic field of the plages, closely related to the very fine structures of "filigrees" type (now studied using the so-called G-band filtergrams), and that of the sunspots which emerge towards a very complex penumbra with multiple components, are not less easy to study. The coronal counterparts are only very often arbitrary considered, including with regard to the heating. Looked in detail, the effective ignorance of these chromospheric layers which however contain a determining part of the magnetism at the origin of the heating of the corona and of its filling and, especially, of all the structure responsible for the eruptions and "flares", is absolutely amazing. Observations of sufficient quality in good cadence and of photometric reproducibility (to allow to consider the current differences in images) are cruelly missing. Ly $\alpha$ observations up to now are almost non-existent beside rocket flights snapshots (or not very convincing when observed with TRACE whose filter is not adequate and the fields of view too reduced).

As it is impossible to measure directly the amplitude of the coronal magnetic field (emission lines are too weak and, moreover, too much widened by the effects of turbulence and Doppler shifts), the systematic study of the chromospheric layers is of great importance and the best line for that is $\operatorname{Ly} \alpha$, which results promise to be rather extraordinary, provided that the filtering of the line is sufficient and the rate (cadence) of measurements high enough. It should be noted that space X-EUV hardly allows the study of the chromosphere (except, to some extent, with the lines of helium with a so particular behavior).

\section{LAIME instrument}

The LAIME Ly $\alpha$ imager consists in a single telescope of $60 \mathrm{~mm}$ useful aperture, the high spectral resolution of $\sim 40 \AA \mathrm{FWHM}$ being obtained with a double filtering between a back covered MgF2 $6 \mathrm{~mm}$ thick entrance window and a classical filter before the CCD. The entrance window reduces significantly the solar flux in the telescope. The instrument has no mechanisms and dimensions are very limited: $100 \times 100 \times 400 \mathrm{~mm}$ (cf. Fig. 4). Furthermore, the thermal control is passive since based on the same successful design 
than TRC and the Helium II telescope of TESIS. It consists in INVAR bars between the primary and secondary mirror supports whose dilatation is compensated by a small slab of aluminum in extra on the secondary support. Such a simple system allows to keep precise dimensions for a temperature range of $\pm 10^{\circ}$ around $20^{\circ}$.

The telescope itself is a Ritchey-Chrétien of focal length $1 \mathrm{~m}$ with a CCD of $1024 \times 1024$ active pixels of $13 \mu \mathrm{m}$ (E2V 47-20 CCD, AIMO, frame transfer, back thinned), allowing a spatial resolution of 5.4" (2.69" per pixel). A higher spatial resolution (2k CCD) is also under discussion to achieve a more appropriate resolution of 2.7 ".

Table 2. LAIME Ly $\alpha$ imager instrument performances.

\begin{tabular}{c|c|c|c|c}
\hline Wavelength $(\mathrm{nm})$ & Field-of-view & $\begin{array}{c}\text { Spatial resolution } \\
(\operatorname{arcsec})\end{array}$ & $\begin{array}{c}\text { Spectral } \\
\text { resolution }(\mathrm{nm})\end{array}$ & Cadence (s) \\
\hline 121.6 & 2.8 solar radii & $\begin{array}{c}5.4 \\
(2.7)\end{array}$ & $\sim 4(\mathrm{FWHM})$ & 10 \\
\hline
\end{tabular}

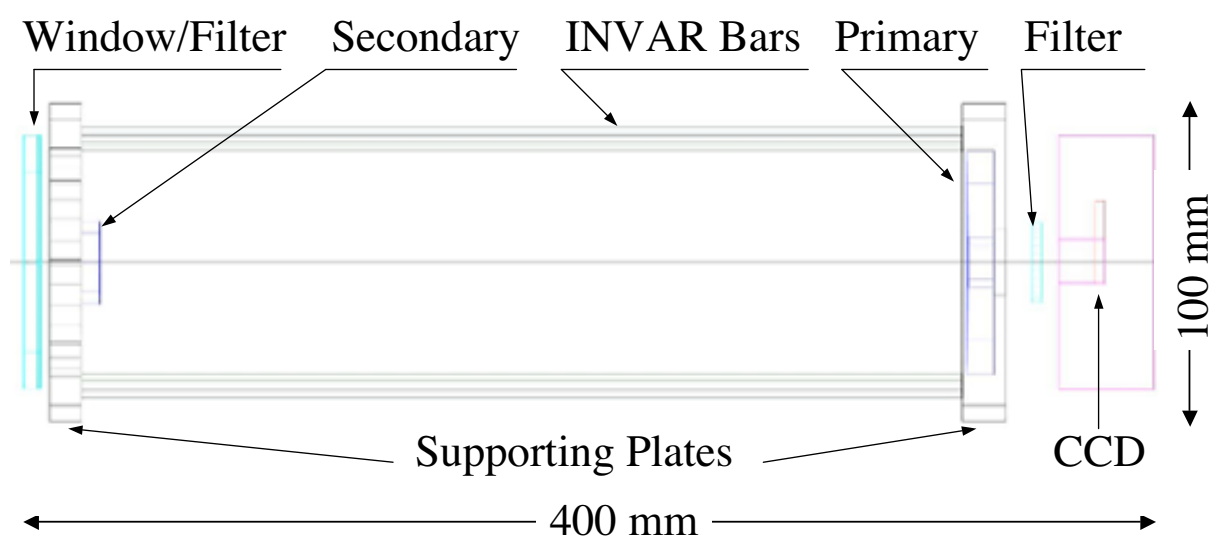

Figure 4. Simplified view of the LAIME instrument showing the telescope with the window/filter (thickness $6 \mathrm{~mm}$ in MgF2), optics, bars in INVAR (4), secondary Ly $\alpha$ filter (thickness $3 \mathrm{~mm}$ in $\mathrm{MgF} 2)$ and $\mathrm{CCD}(1 \mathrm{k} \times 2 \mathrm{k}$, frame transfer, back thinned). Proportions are preserved.

\section{Conclusion}

The needs for a very high-resolution mission with high telemetry throughput, large spectral coverage and continuity of observations, have never been so strongly expressed by the solar community. Accordingly, a mission building on STEREO, SDO and SOLAR$\mathrm{B}$, and filling the current observational gap in the UV and FUV, high chromosphere and transition zone, is proposed for the next solar maximum in 2012, well before the Solar Orbiter first data which are not expected before end 2018 (for a flight in May 2015). This venture could materialize in a collaborative mission around SOLARNET between CNES, India and China (CNES new mission cycle could be decided as early as 2007 for a flight in 2012). As well, and although not so timely with the solar cycle, an ESA Cosmic Vision 2015-2016 proposal is planned to complement the Solar Orbiter plasma measurements. In the mean time, smaller and more specialized initiatives like LAIME on TESIS/CORONAS, will hopefully provide results of the critical chromosphere-corona 
interface through $\operatorname{Ly} \alpha$ line observations of the magnetic field topology, variability and dynamics.

\section{Acknowledgements}

The mission proposed around SOLARNET is based on contributions inherited from the SIMURIS and SPI missions' proposals to ESA and, in that respect, we are particularly grateful to M. Derrien, A. Cacciani, F. Clette, R. Kariyappa, J. Porteneuve, A. Preumont, and E. Quémerais. We also acknowledge CNES instances for the R \& D support of the SOLARNET breadboard and PHD fellowships, and are indebted to the French Embassy of Beijing for financing a PHD on this program. Finally, we are thankful to B. Schmieder, S. Turck-Chieze, R. Lallement, E. Quémerais, P. Boumier, P. Lamy, J.-M., Malherbe, J.-C. Noëns, J.-F. Hochedez, D. Berghmans, S. Fineschi, W. Curdt, E. Marsch, V. Bothmer, P. Cargill, R. Harrison, S. Hasan, R. Kariyappa, G. Ai, J. Klimchuk, A. Vourlidas and T. Tarbell, for contributions and discussions to both SOLARNET and LAIME.

\section{References}

Brekke, P. 1999, Observations of Transition Region Plasma, Solar Phys., 190, 379-408

Damé, L., Cladé, S. \& Zhao, B. 2004, Solar Full Field Interferometric Imaging with 3 Telescopes, in: B. Warmbein (ed.), 5th International Conference on Space Optics, ESA-SP, 554, 373379

Damé, L. 2003, A Solar Imaging Interferometer: the SOLARNET Breadboard at Paris/Meudon Observatory, in: J.C. Hénoux, C. Fang \& N. Vilmer (eds.), Second French-Chinese Meeting on Solar Physics, Understanding Active Phenomena: Progress and Perspectives, 277-286

Damé, L. \& Derrien, M. 2002, The Solar Physics and Interferometry Mission (SPI), in: L. Damé \& E. Marsch (eds.), Current and Future High Resolution In-situ and Remote Sensing Solar Physics Missions, Adv. Space Res., 29(12), 2075-2082

Damé, L., Derrien, M., Kozlowski, M., Perrot, S. \& Preumont, A. 2002, Technologies for Solar Interferometry in Space, in: L. Damé \& E. Marsch (eds.), Current and Future High Resolution In-situ and Remote Sensing Solar Physics Missions, Adv. Space Res., 29(12), 2061-2073

Dere, K.P., Bartoe, J.-D., Brueckner, G.E., Cook, J.W. \& Socker, D.G. 1987, Discrete Subresolution Structures in the Solar Transition Zone, Solar Phys., 114, 223-237

Filippov, I. \& Koutchmy, S. 2000, Solar Phys., 196, 311

Fu, X., Damé, L., Kozlowski, M., Ziaei, N., Cladé, S., Montaron, C. \& Maury, V. 2006, The SOLARNET 3-Telescopes Breadboard Development, in: C. Fang \& B. Schmieder (eds.), Third French-Chinese Meeting on Solar Physics, Beijing World Publishing Corporation, Kluwer Adademic Publishers, to be published

Klimchuk, J. 2006, Impulsive coronal heating in tangled magnetic fields, these proceedings

Millard, A.A., Lemaire, P. \& Vial, J.C. 2005, EUV Imaging Spectrometer for the SOLARNET Mission, Adv. in Space Res., 36, 1411-1414

Thorne, A.P. 1992, IFTS: the Imaging Fourier Transform Spectrometer, in: L. Damé \& T.D. Guyenne (eds.), ESA Workshop on Solar Physics and Astrophysics at Interferometric Resolution, ESA SP-344, 191-195 